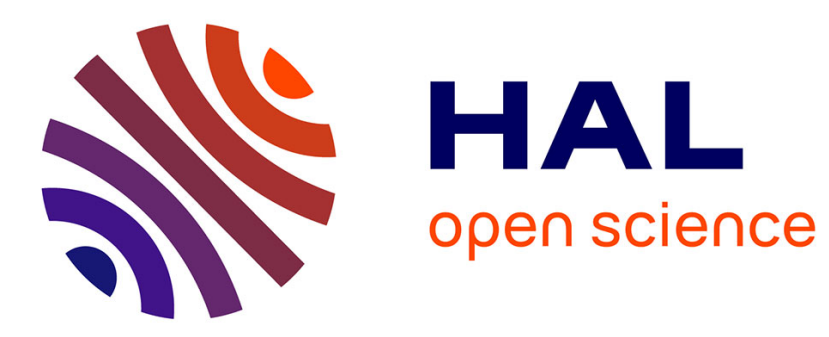

\title{
On a conjecture of Helleseth
}

Yves Aubry, Philippe Langevin

\section{- To cite this version:}

Yves Aubry, Philippe Langevin. On a conjecture of Helleseth. Lecture Notes in Computer Science, 2013, 8080, pp.113-118. 10.1007/978-3-642-40663-8_12 . hal-00769143

\section{HAL Id: hal-00769143 \\ https://hal.science/hal-00769143}

Submitted on 28 Dec 2012

HAL is a multi-disciplinary open access archive for the deposit and dissemination of scientific research documents, whether they are published or not. The documents may come from teaching and research institutions in France or abroad, or from public or private research centers.
L'archive ouverte pluridisciplinaire HAL, est destinée au dépôt et à la diffusion de documents scientifiques de niveau recherche, publiés ou non, émanant des établissements d'enseignement et de recherche français ou étrangers, des laboratoires publics ou privés. 


\title{
ON A CONJECTURE OF HELLESETH
}

\author{
YVES AUBRY AND PHILIPPE LANGEVIN
}

\begin{abstract}
We are concern about a conjecture proposed in the middle of the seventies by Hellesseth in the framework of maximal sequences and theirs crosscorrelations. The conjecture claims the existence of a zero outphase Fourier coefficient. We give some divisibility properties in this direction.
\end{abstract}

\section{Two Conjectures of Helleseth}

Let $L$ be a finite field of order $q>2$ and characteristic $p$. Let $\mu$ be the canonical additive character of $L$ i.e. $\mu(x)=\exp (2 i \pi \operatorname{Tr}(x) / p)$ where $\operatorname{Tr}$ is the trace function with respect to the finite field extension $L / \mathbb{F}_{p}$. The Fourier coefficient of a mapping $f: L \rightarrow L$ is defined at $a \in L$ by

$$
\widehat{f}(a)=\sum_{x \in L} \mu(a x+f(x)) .
$$

The distribution of these values is called the Fourier spectrum of $f$. Note that when $f$ is a permutation the phase Fourier coefficient $\widehat{f}(0)$ is equal to 0 .

The mapping $f(x)=x^{s}$ is called the power function of exponent $s$, and it is a permutation if and only if $(s, q-1)=1$. Moreover, if $s \equiv 1 \bmod (p-1)$ the Fourier coefficients of $f$ are rational integers. Helleseth made in [3] two "global" conjectures on the spectra of power permutations. The first claims the vanishing of the quantity (related to Dedekind determinant, see [9])

$$
\mathfrak{D}(f)=\prod_{a \in L^{\times}} \widehat{f}(a) .
$$

Conjecture 1 (Helleseth). Let $L$ be a field of cardinal $q>2$. If $f$ is a power permutation of exponent $s \equiv 1 \bmod (p-1)$ then $\mathfrak{D}(f)=0$.

For $p=2$, it generalizes Dillon's conjecture (see [2]) which corresponds to the case $s=q-2 \equiv-1 \bmod (q-1)$, and known to be true because it is related to the vanishing of Kloosterman sums and the class number $h_{q}$ of the imaginary quadratic number field $\mathbb{Q}(\sqrt{1-4 q})$ (see $[5,8]$ ). Note also that in odd characteristic the Kloosterman sums do not vanish (see [7]) except if $p=3$ (see [5]).

The second conjecture deals with the number of values in the spectrum of a power permutation.

Conjecture 2. If $\left[L: \mathbb{F}_{p}\right]$ is a power of 2 then the spectrum of a power function takes at least four values.

In this note, we prove some results concerning the divisibility properties of the Fourier coefficients of power permutations in connection with Conjecture 1. Our results can be seen as a proof "modulo $\ell$ " of Conjecture 1 for certain primes $\ell$. 
TABLE 1. An example of Walsh spectrum having only one Walsh coefficient equal to zero (see $[6]$ ).

\begin{tabular}{lcccccccccc}
\hline Walsh & -48 & -44 & -40 & -36 & -32 & -28 & -24 & 20 & -16 & -12 \\
\hline mult. & 5 & 30 & 85 & 70 & 115 & 100 & 31 & 62 & 20 & 10 \\
\hline \hline Walsh & 0 & 8 & 16 & 20 & 24 & 28 & 32 & 36 & 40 & 44 \\
\hline mult. & 1 & 5 & 25 & 20 & 85 & 90 & 90 & 80 & 50 & 50 \\
\hline
\end{tabular}

\section{BoOleAn FUnCtion CASE}

In this section, we assume $p=2$. In [10], the second author has computed the Fourier spectra of power permutations for all the fields of characteristic 2 with degree less or equal to 25 without finding any counter-example to the above conjectures. More curiously, if we denote by nbz $(s)$ the number of Fourier coefficients of the power function of exponent $s$ equal to zero then the numerical experience suggests that:

$$
\operatorname{nbz}(s) \geq \operatorname{nbz}(-1)=h_{q} .
$$

At this point, it is interesting to notice that Helleseth's conjecture can not be extended to the set of all permutations. Indeed, let $m$ be a positive integer and let $g: \mathbb{F}_{2}^{m} \rightarrow \mathbb{F}_{2}$ be a Boolean function in $m$ variables. One defines the Walsh coefficient of $g$ at $a \in \mathbb{F}_{2}^{m}$ by :

$$
g^{\mathcal{W}}(a)=\sum_{x \in \mathbb{F}_{2}^{m}}(-1)^{a . x+g(x)} .
$$

Identifying $L$ with the $\mathbb{F}_{2}$-vector space $\mathbb{F}_{2}^{m}$, the Boolean function $g$ has a trace representation i.e. there exists a mapping $f: L \rightarrow L$ such that $g(x)=\operatorname{Tr}_{L}(f(x))$ for all $x$ in $L$. Of course, the trace representation is not unique. Moreover, if $g$ is balanced then $g$ can be represented by a permutation of $L$. In all the cases, the Walsh spectrum of $g$ and the Fourier spectrum of $f$ are identical.

In [6], an example of a ten variables Boolean function with a very atypical Walsh spectrum (see Tab. 1) is given. This Boolean function is balanced and its Walsh coefficients vanish only once. This numerical example, say $g$, implies the existence of a permutation $f$ of $\mathbb{F}_{1024}$ (not a power permutation) such that

$$
g(x)=\operatorname{Tr}_{\mathbb{F}_{1024}} f(x),
$$

whence the Fourier spectrum of $f$ is equal to the Walsh spectrum of $g$, and thus $\sum_{x \in \mathbb{F}_{1024}} \mu(a x+f(x)) \neq 0$ for all $a \in \mathbb{F}_{1024}^{\times}$.

A possible generalization of the conjecture of Helleseth, proposed by Leander, could be the following one:

Conjecture 3. If $f$ is a permutation of $L$ then $\prod_{\lambda \in L^{\times}} \mathfrak{D}(\lambda f)=0$.

Note that Conjecture 2 is know to be true in characteristic 2 since recent works of Daniel Katz in [4] and Tao Feng in [11]. In order to complete this short conjecture tour, we recall to the reader the main global conjecture of the domain due to Sarwate and which is still open

Conjecture 4. If $f$ is a power permutation of $L$ where $\left[L: \mathbb{F}_{2}\right]$ is even then $\sup _{a \in L} \widehat{f}(a) \geq 2 \sqrt{q}$. 
In the sequel, if $\lambda \in L$ then we denote by $\widehat{f_{\lambda}}(a)$ the Fourier coefficient of $x \mapsto$ $\lambda f(x)$. If $f$ is a power permutation of exponent $s$, denoting by $t$ the inverse of $s$ modulo $q-1$, for all $y \in L^{\times}$, we have :

$$
\widehat{f_{\lambda}}(a)=\sum_{x \in L} \mu\left(\lambda x^{s}+a x\right)=\sum_{x \in L} \mu\left(\lambda y^{s} x^{s}+a x y\right)=\widehat{f}\left(a \lambda^{-t}\right) .
$$

Hence, one of the specifics of power permutations among the permutations of $L$ is that the spectrum of $\lambda f$ does not depend on $\lambda \in L^{\times}$.

We conclude this section by giving a divisibility result. Recall that a function $f$ defined over a field $L$ of characteristic 2 is said to be almost perfect nonlinear if for all $u \in L^{\times}$the derivative $x \mapsto f(x+u)+f(x)$ is two-to-one. It is for example the case of $f(x)=x^{3}$ over any field $L$ and of $f(x)=x^{-1}$ when $\left[L: \mathbb{F}_{2}\right]$ is odd.

Proposition 1. Let $f$ be a power permutation over a field $L$ of characteristic two and cardinal $q \not \equiv 2,4 \bmod 5$. If $f$ is almost perfect nonlinear then there exists $a \in L^{\times}$such that $\widehat{f}(a) \equiv 0 \bmod 5$.

Proof. It is well-known (see [1]) that an APN function $f$ satisfies

$$
\sum_{\lambda \in L^{\times}} \sum_{a \in L} \widehat{f_{\lambda}}(a)^{4}=2 q^{3}(q-1) .
$$

Since the spectrum of $\lambda f$ does not depend on $\lambda \in L^{\times}$, it implies that:

$$
\sum_{a \in L} \widehat{f_{\lambda}}(a)^{4}=2 q^{3} .
$$

Assuming $\mathfrak{D}(f) \not \equiv 0$ mod 5 , we get the congruence $q-1 \equiv 2 q^{3} \bmod 5$ implying $q \equiv 2,4 \bmod 5$.

\section{HYPERPLANE SECTION}

The key point of view of this note is to consider the number, say $N_{n}(u, v)$, of solutions in $L^{n}$ of the system

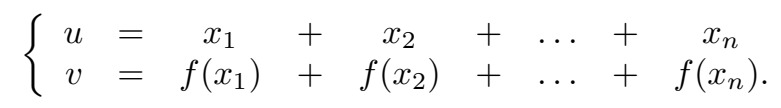

Using characters counting principle, we can write:

$$
\begin{aligned}
q^{2} N_{n}(u, v) & =\sum_{x_{1}, x_{2}, \ldots, x_{n}} \sum_{\beta \in L} \sum_{\alpha \in L} \mu_{\beta}\left(\sum_{i} f\left(x_{i}\right)+v\right) \mu_{\alpha}\left(\sum_{i} x_{i}+u\right) \\
& \left.=\sum_{\beta} \sum_{\alpha}\left(\sum_{y} \mu(\beta f(y)+\alpha y)\right)\right)^{n} \mu(\alpha u+\beta v) \\
& =\sum_{\beta} \sum_{\alpha} \widehat{f_{\beta}}(\alpha)^{n} \mu(\alpha u+\beta v) \\
& =\sum_{\alpha} \widehat{1}(\alpha)^{n} \mu(\alpha u)+\sum_{\beta \neq 0} \sum_{\alpha} \widehat{f_{\beta}}(\alpha)^{n} \mu(\alpha u+\beta v) \\
& =q^{n}+\sum_{\alpha \neq 0} \sum_{\beta \neq 0} \widehat{f_{\beta}}(\alpha)^{n} \mu(\alpha u+\beta v)
\end{aligned}
$$


Lemma 1. Assuming the Fourier coefficients of $\lambda f, \lambda \in L$, are integers. Let $\ell$ be a prime such that $\prod_{\lambda \in L^{\times}} \mathfrak{D}(\lambda f) \not \equiv 0 \bmod \ell$. Then

$$
q^{2} N_{\ell-1}(u, v) \equiv 1+\left(q \delta_{0}(u)-1\right)\left(q \delta_{0}(v)-1\right) \bmod \ell
$$

where $\delta_{a}(b)$ is equal to 1 if $b=a$ and 0 otherwise.

Proof. By the Fermat's little Theorem, we have the congruence

$$
\widehat{f_{\lambda}}(a)^{\ell-1} \equiv 1-\delta_{0}(a) \bmod \ell
$$

Hence

$$
\begin{aligned}
q^{2} N_{\ell-1}(u, v) & =q^{\ell-1}+\sum_{\alpha \neq 0} \sum_{\beta \neq 0} \widehat{f_{\beta}}(\alpha)^{\ell-1} \mu(\alpha u+\beta v) \\
& \equiv 1+\sum_{\alpha \neq 0} \sum_{\beta \neq 0} \mu(\alpha u+\beta v) \quad \bmod \ell
\end{aligned}
$$

and we conclude remarking that $\sum_{\alpha \in L^{\times}} \mu(\alpha u)=q \delta_{0}(u)-1$.

\section{Divisibility of Fourier COEFFICIENTS}

In [3], it is proved that for the exponents $s \equiv 1 \bmod (p-1)$, the Fourier coefficients are multiple of $p$. In this section, we are interested in divisibility properties modulo a prime $\ell \neq p$.

Assuming that the Fourier coefficients of a mapping $f$, not necessary a power function, are rational integers, we can see that if 3 does not divide $\mathfrak{D}(f)$ then we have necessarily $q \equiv 2 \bmod 3$. Indeed, using Parseval relation, we can write

$$
1 \equiv q^{2}=\sum_{a \in L}|\widehat{f}(a)|^{2}=\sum_{a \in L} \widehat{f}(a) \equiv q-1 \quad \bmod 3
$$

Theorem 1. Let $f$ be the power function of exponent $s$. If $s=1 \bmod (p-1)$ is coprime with $q-1$ then $\mathfrak{D}(f) \equiv 0 \bmod 3$.

Proof. Suppose that $\mathfrak{D}(f) \not \equiv 0 \bmod 3$. Applying Lemma 1 with $\ell=3$, we get

$$
\forall u \in L^{\times}, \quad \forall v \in L^{\times}, \quad N_{2}(u, v) \not \equiv 0 \quad \bmod \ell .
$$

To complete the proof we prove the existence of a pair $(u, v)$ of nonzero elements such that $N_{2}(u, v)=0$. Let us fix $u=1$, the $v$ 's such that $N_{2}(1, v)>0$ are in the image of $L$ by the mapping $x \mapsto(1-x)^{s}+x^{s}$, if $x$ is a preimage of $v$ then $1-x$ is an other one except if $p=2$ and $v=2(1 / 2)^{s}$. Thus, if $q>3$, there exists $v \in L^{\times}$ without preimage i.e. $N_{2}(1, v)=0$.

Proposition 2. Let $f$ be a power permutation of exponent $s \equiv 1 \bmod (p-1)$. If $\left[L: \mathbb{F}_{p}\right]$ is a power of a prime $\ell$ and $p \not \equiv 2 \bmod \ell$ then $\mathfrak{D}(f) \equiv 0 \bmod \ell$. 
Proof. The Frobenius automorphism acts on the solutions of the system (6) with $u=0, v=1$. Since $s \equiv 1 \bmod (p-1)$, the system has no $\mathbb{F}_{p^{-} \text {-solutions, thus }}$ $N_{\ell-1}(0,1) \equiv 0 \bmod \ell$. On the other hand, by Lemma 1 , if $\mathfrak{D}(f) \not \equiv 0 \bmod \ell$ then

$$
q^{2} N_{\ell-1}(0,1) \equiv 2-q \equiv 2-p \quad \bmod \ell .
$$

\section{REFERENCES}

[1] Florent Chabaud and Serge Vaudenay. Links between diffrential and linear cryptanalysis. Eurocrypt 94, 950:356-365, 1994.

[2] John F. Dillon. Elementary Hadamard Difference Sets. PhD thesis, Univ. of Maryland, 1974.

[3] Tor Helleseth. Some results about the cross-correlation function between two maximal linear sequences. Discrete Math., 16(3):209-232, 1976.

[4] Daniel J. Katz. Weil sums of binomials, three-level cross-correlation, and a conjecture of Helleseth. J. Comb. Theory, Ser. A, 119(8):1644-1659, 2012.

[5] Nicholas Katz and Ron Livné. Sommes de Kloosterman et courbes elliptiques universelles en caractéristiques 2 et 3. C. R. Acad. Sci. Paris Sér. I Math., 309(11):723-726, 1989.

[6] Selçuk Kavut, Subhamoy Maitra, and Melek D. Yücel. Search for boolean functions with excellent profiles in the rotation symmetric class. IEEE Transactions on Information Theory, 53(5):1743-1751, 2007.

[7] Kononen Keijo, Rinta-Aho Marko, and Vaanainen Keijoe. On integer value of Kloosterman sums. IEEE trans. info. theory, 2010.

[8] Gilles Lachaud and Jacques Wolfmann. Sommes de Kloosterman, courbes elliptiques et codes cycliques en caractéristique 2. C. R. Acad. Sci. Paris Sér. I Math., 305:881-883, 1987.

[9] Serge Lang. Cyclotomic fields I and II, volume 121 of Graduate Texts in Mathematics. Springer-Verlag, New York, second edition, 1990. With an appendix by Karl Rubin.

[10] Philippe Langevin. Numerical projects page, 2007. http://langevin.univ-tln.fr/project/ spectrum.

[11] Feng Tao. On cyclic codes of length $2^{2^{r}}-1$ with two zeros whose dual codes have three weights. Designs, Codes and Cryptography, 62(3), 2012.

Institut de Mathématiques de Toulon, Université du Sud Toulon-Var, France and Institut de Mathématiques de Luminy, Marseille, France

E-mail address: yves.aubry@univ-tln.fr

Institut de Mathématiques de Toulon, Université du Sud Toulon-Var, France

E-mail address: langevin@univ-tln.fr 